\title{
The Effect of Magnetized Water on the Growth and Physiological Conditions of Moringa Species under Drought Stress
}

\author{
Md. Mahadi Hasan, Hesham F. Alharby, Abdulrahaman S. Hajar, \\ Khalid Rehman Hakeem*, Yahya Alzahrani \\ Department of Biological Sciences, Faculty of Science, King Abdulaziz University, \\ Jeddah, Saudi Arabia
}

Received: 21 December 2017

Accepted: 27 February 2018

\begin{abstract}
Magnetic water technology is supposed to be an eco-friendly tool used for alleviating drought stress in the agricultural sector. The present study investigates the role of magnetized water on the two Moringa species (Moringa oleifera and Moringa peregrina) under drought stress. The experiments were conducted in a greenhouse to compare three watering regimes, including, 100\% field capacity (FC, as Control), $50 \%$ field capacity (FC, as moderate drought stress), and 20\% field capacity (FC, as severe drought stress), and two water treatments (normal water and magnetic water). Significant reductions in plant height, leaf area, relative water content (RWC), chlorophyll and ion contents, assimilation rate (A), transpiration (E), stomatal conductance (gs), water use efficiency (WUE), and vapor pressure deficit (VPD) were observed during the drought stress. M. oleifera and M. peregrina seedlings when treated with MW during SS level, exhibited 13.09\%, 21.1\%, 22.6\%, 23\%, and 10.7\%,15.2\%,12.9\% ,13.19\% decreases in $\mathrm{Chl} \mathrm{a,} \mathrm{Chl} \mathrm{b,} \mathrm{Chl} \mathrm{(a+b)} \mathrm{and} \mathrm{carotenoids} \mathrm{contents} \mathrm{respectively,} \mathrm{as} \mathrm{compared} \mathrm{to} \mathrm{control} \mathrm{seedlings.}$ The M. oleifera and M. peregrina seedlings exposed to drought stress (MS, SS) showed $1.26 \%$, $3.79 \%$, and $1.2 \%, 3.7 \%$ decreases in $\mathrm{F}_{\mathrm{v}} / \mathrm{F}_{\mathrm{m}}$ under MWT. The drought-stressed seedlings treated with magnetic water (MW) recovered from growth inhibition, chlorosis, and ion disruption. Magnetic water (MW) improved the $\mathrm{Na}^{+} / \mathrm{K}^{+}$ratio by lowering the accumulation of $\mathrm{Na}^{+}$ions. Hence, the application of magnetized water (MW) mitigated the adverse effects of drought in the Moringa species.
\end{abstract}

Keywords: magnetic water, Fv/Fm, ion content, photosynthesis, abiotic stress, leaf gas exchange

\section{Introduction}

As the global population expands, the economic, agricultural, industrial, and energy sectors also grow,

*e-mail: kur.hakeem@gmail.com due to which water consumption and demand has vastly increased. Anthropogenic activities are leading toward an increase of greenhouse gases, which are responsible for global climate change. Consequently, drought has become more common and severely occurred due to all of these factors [1-3]. Drought is one of the serious problems that has withheld global crop 
production, considered as a major abiotic stress that has affected plant growth rate and development [3-5]. Due to changing climatic conditions, water shortages have become more apparent not just in arid regions, but humid regions as well [6]. Unequal rainfall patterns have been found to limit plant growth [7]. As water is required for almost all biochemical, physiological, and morphological plant processes, its shortage is harmful for their normal growth and development [8]. Drought stress affects various metabolic activities, including photosynthesis in the plants [9-10]. The leaf gas exchange traits such as assimilation (A), transpiration (E), stomatal conductance (gs), water use efficiency (WUE), vapor pressure deficit (VPD), and maximum quantum efficiency of the photosystem II were known to decrease under drought stress [11-14]. Reduction of the photosynthetic electron chain occurred due to stomatal closure and a decrease in $\mathrm{CO}_{2}$ fixation under drought stress [15].

In recent years, various approaches have been used to alleviate the drought stress among agricultural crops. These include nutritional management strategies, the application of chemical and biological amendments (including mycorrhizal applications), and the use of robotics and biosensors [15-16]. Some modern technologies have been used for environmental management, including GIS [15] and nanotechnology [17-22]. Magnetic water (MW) technology is one of the environmentally friendly and cost-effective techniques, recently used in agricultural fields to result in better crop growth and yield [23]. MW is generated by treating normal water with a magnetic field, resulting in the rearrangement of water structure into a new hexagonal form in various ways [23]. Many researchers have reported the beneficial effects of MW in the agricultural sector [16, 24-26].

$M$. oleifera and $M$. peregrina are the two common species among the family of the Moringaceae. $M$. oleifera is regarded as a multipurpose tree and shrub species and it is used as a food, fodder, medicine, fencing, gum, and firewood, as well as a coagulant to treat polluted water [27]. M. peregrina is also considered a medicinally important plant due to its antioxidant, antifungal, and antibacterial properties [28].

Since MW has been reported to have positive effects on plant growth and physiology, it was hypothesized that this technique may also improve plant physiology and growth characteristics under drought stress. Subsequently, some information is available about the application of MW on plants under drought stress; a broader study, however, is absent regarding the effects of drought stress on the growth and physiology of Moringa species under MWT. The present study was carried out to assess the effects of drought stress on the growth and some physiological traits of the Moringa species under magnetic water treatment (MWT). The results of this study may provide information for improving the irrigation methods and management through MWT - especially for the production of Moringa species under drought stress.

\section{Materials and Methods}

\section{Experimental Materials and Setup}

The experiment was carried out at a greenhouse in King Abdulaziz University (KAU), Jeddah, Saudi Arabia. The seeds of two Moringa species (M. oleifera and $M$. peregrina) were collected from the Abha region, Saudi Arabia. Seeds were collected in sealed plastic bags and transferred to the laboratory at KAU. The seeds were sown in the pot under randomized completely block design (RCBD). All seeds were sown on the same day. The soil was sandy loam, which was mixed with peat moss and compost (1:1:1) vigorously. The pots were kept under greenhouse-controlled conditions (light/ dark regime about $12 / 12 \mathrm{~h}$; temp., $25 / 15 \pm 30^{\circ} \mathrm{C}$; relative humidity (RH) $30-50 \%$ ). A magnetic instrument was used (150d magnetic technologies L.L.C) with power of $30 \mathrm{mT}$ for magnetizing the water by following the methods of Selim and El-Nady (2011) [29]. Half of the pots were irrigated with normal tap water and remaining pots were irrigated with the MW.

\section{Field Capacity Measurements}

Water-holding capacity or field capacity of soil was determined according to the gravimetric method with slight modifications [30]. The experiment was done with five replicates. Soil samples were collected from the source of used soil in the experiment. First, soil was air dried and ground to pass through a $5 \mathrm{~mm}$ sieve. The bottoms of small cylindrical pots were covered with paper and taped without soil. Each of the pots are loaded with $300 \mathrm{~g}$ soil (by compression) and weighed. All pots were settled in the tray and filled with water until saturation. The tray was allowed $3 \mathrm{~h}$ for saturation and then all pots were transferred to quartz soil intended for drainage and filtering for $2 \mathrm{~h}$. The pots were cleaned and weighed the saturated soil again. Then all saturated soil was oven dried at $105^{\circ} \mathrm{C}$ for $24 \mathrm{~h}$ and dry weight was calculated by measuring the oven-dried soil. Soil field capacity was measured using the following formula:

$$
\begin{gathered}
\text { Field capacity }(\mathrm{FC} \%)= \\
\frac{\text { Saturated soil weight }- \text { Dry weight of soil }}{\text { Dry weight of soil }} \times 100 \%
\end{gathered}
$$

\section{Drought Stress Treatments}

Plants were irrigated daily with normal and magnetic water up to 60 days for the plant establishment. After the plant establishment, Moringa species were subjected to drought treatment including 100\% FC 
(control), 50\% FC (moderate stress, MS), and 20\% FC (severe stress, SS) for 30 days. The plant sample was collected during harvest time at 90 days.

\section{Growth Conditions}

Growth parameters were summarized by the analysis of plant height, leaf number, shoot length, root length, internodes distance, leaf area, and fresh and dry weights. Plant height $(\mathrm{cm})$ and internode distances $(\mathrm{cm})$ were measured from the surface to the top of the plant at 5DAT to 30 DAT. The plant parts (leaf, stem, root) were dried at $70^{\circ} \mathrm{C}$ for $72 \mathrm{~h}$ and dry weight $(\mathrm{g})$ were obtained.

\section{Leaf Area and Relative Water Content}

A leaf area meter (LICOR-3000A, USA) was used for measuring leaf area. For calculating the relative water content (RWC), leaf discs were obtained from seedlings and weighed, and fresh weight (FW) was obtained. The leaf discs were taken and floated on distilled water. After $8 \mathrm{~h}$, surface water was removed using paper towels and turgid weight (TW) was obtained. Finally, the leaves were dried at $80^{\circ} \mathrm{C}$ for $48 \mathrm{~h}$ and dry weight (DW) was obtained. The RWC was calculated by the following formula:

$$
\mathrm{RWC}(\%)=\frac{\mathrm{FW}-\mathrm{DW}}{\mathrm{TW}-\mathrm{DW}} \times 100
$$
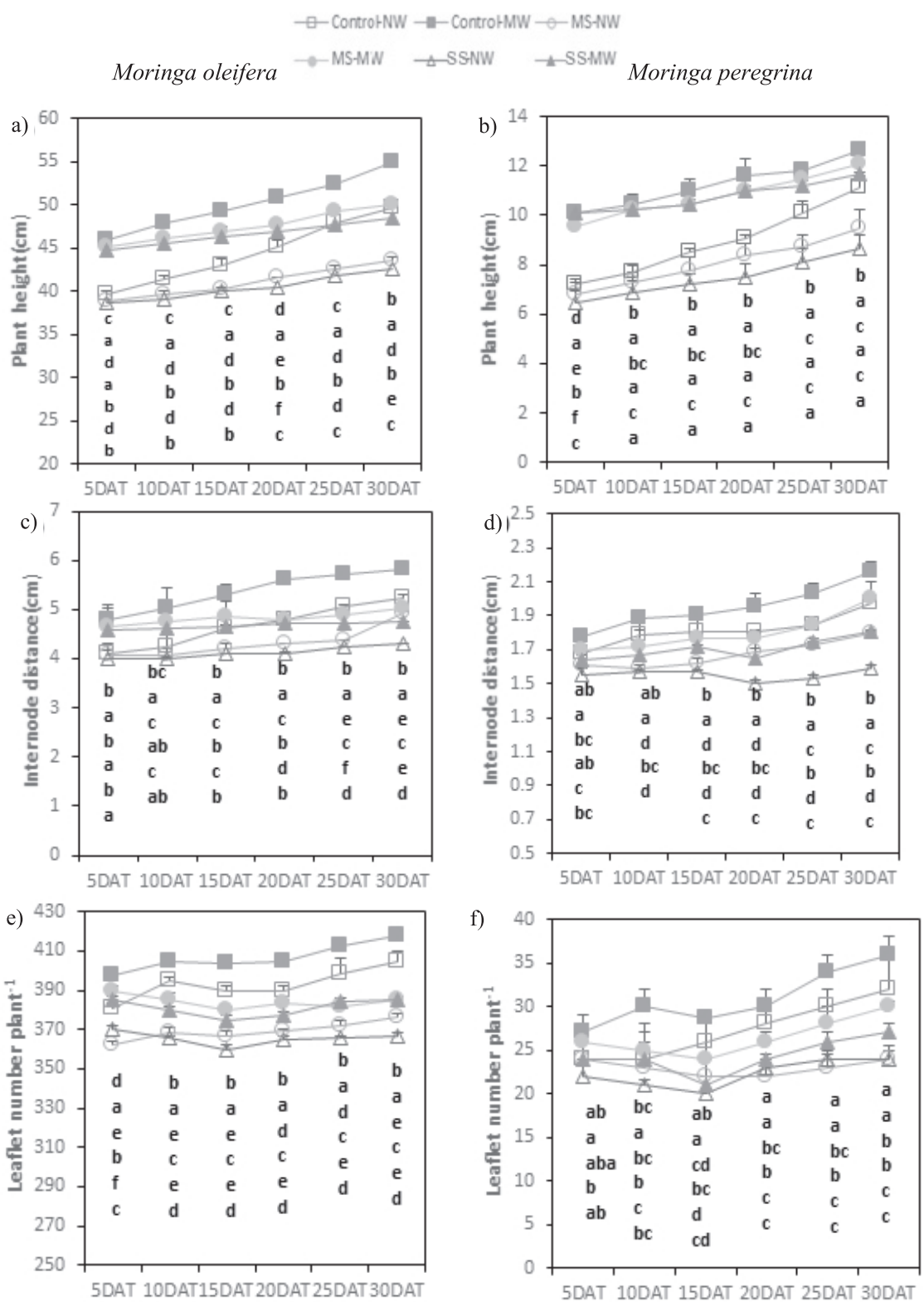

Fig. 1. Effect of magnetic water (MW) on plant height (a, b), internode distances (c, d), and leaflet number (e, f) of two Moringa species (Moringa oleifera, Moringa peregrina) seedlings under drought stress at 5 to 30 days after treatment; vertical bar represents the standard deviation, and different letters below the series denote statistically significant $(\mathrm{P} \leq 0.05)$ differences among treatments. 


\section{Leaf Gas Exchange and Fv/Fm Ratio}

Leaf gas exchanges were measured by the CIRAS 3 portable photosynthesis system (PP systems, USA). The leaf gas exchange parameters included assimilation rate $\left(\mathrm{A}, \mathrm{CO}_{2} \mathrm{~m}^{-2} \mathrm{~s}^{-1}\right)$, stomatal conductance ( $\mathrm{gs}$, mol $\left.\mathrm{CO}_{2} \mathrm{~m}^{-1} \mathrm{~s}^{-1}\right)$, transpiration rate $\left(\mathrm{E}, \mathrm{mmol} \mathrm{CO} 2 \mathrm{~m}^{-2} \mathrm{~s}^{-1}\right)$, vapor pressure deficit (VPD, $\mathrm{kPa}$ ), and water use efficiency (WUE, $\mu \mathrm{molCO}_{2} \mathrm{mmol}^{-1} \mathrm{H}_{2} \mathrm{O}$ ). The young, fully developed and healthy leaves were selected for measurement. The leaf was measured between 12:00 and 14:00 $\mathrm{h}$ (solar radiation is at maximum intensity) in ambient conditions. Three readings from each treatment were taken carefully out on randomly chosen leaves.

The maximum quantum yield of PSII photochemistry $(\mathrm{Fv} / \mathrm{Fm})$ was measured during the experiment at the altered period and at the end of relief on the same leaves used for gas exchange measurements. The leaves of both species were measured after dark adaption of leaf for $30 \mathrm{~min}$ with leaf cuvette. A CIRAS 3 portable photosynthesis system (PP systems, USA) machine was used to generate the data.

\section{Chlorophyll Content}

Chlorophyll contents or photosynthetic pigments were determined using Arnon (1949) [31] methods by homogenizing leaf samples $(0.5 \mathrm{~g})$ with 10 milliliters $(\mathrm{ml})$ of acetone $(80 \% \mathrm{v} / \mathrm{v})$, followed by centrifuging at $5,000^{\prime} \mathrm{g}$ for $10 \mathrm{~min}$. The absorbance was measured with a UV-visible spectrophotometer (VIS-723G) at 663, 645, and $470 \mathrm{~nm}$, respectively, according to Lichtenthaler and Wellburn (1983) [32].

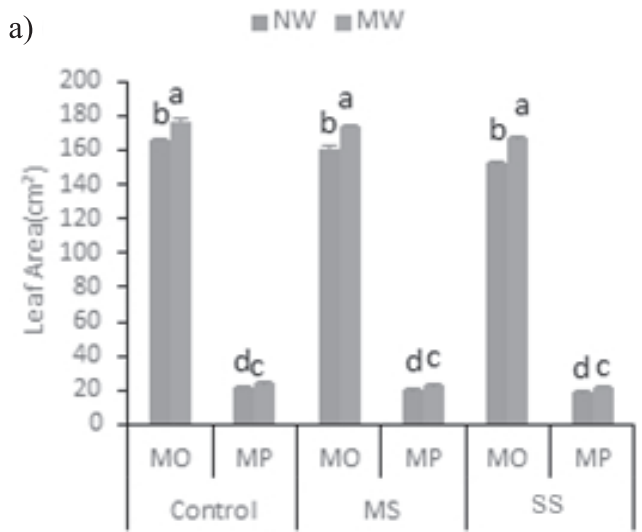

b) $\square$ NW $=M W$

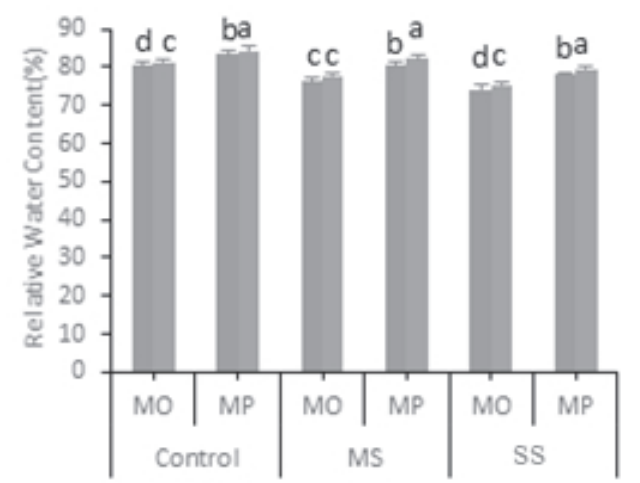

Fig. 2. Leaf area a) and relative water content b) of the two Moringa species (Moringa oleifera, MO; Moringa peregrina, MP) under drought stress; dissimilar letters with mean are significantly different at $\mathrm{p} \leq 0.05$ level of significance by applying Fisher's LSD test.

Table 1. Effect of magnetic water on the fresh and dry weights of root, stem, and leaf under drought stress.

\begin{tabular}{|c|c|c|c|c|c|c|c|}
\hline & \multirow{2}{*}{$\begin{array}{c}\text { Treatment } \\
\text { Combinations }\end{array}$} & \multicolumn{2}{|c|}{ Root } & \multicolumn{2}{|c|}{ Shoot } & \multicolumn{2}{|c|}{ Leaf } \\
\hline & & FW & DW & FW & DW & FW & DW \\
\hline \multirow{6}{*}{$\begin{array}{c}\text { Moringa } \\
\text { oleifera }\end{array}$} & Control+NW & $13.44 \pm 1.3 \mathrm{a}$ & $2.54 \pm 0.13 \mathrm{ab}$ & $13.1 \pm 0.95 \mathrm{bc}$ & $2.3 \pm 0.2 \mathrm{ab}$ & $24.06 \pm 0.2 b$ & $4.06 \pm 0.04 \mathrm{a}$ \\
\hline & Control+MW & $14.3 \pm 1.41 \mathrm{a}$ & $2.62 \pm 0.1 \mathrm{a}$ & $14.53 \pm 1.03 \mathrm{a}$ & $2.45 \pm 0.1 \mathrm{a}$ & $25.06 \pm 1.06 \mathrm{a}$ & $4.13 \pm 0.13 \mathrm{a}$ \\
\hline & $\mathrm{MS}+\mathrm{NW}$ & $12.6 \pm 0.8 \mathrm{ab}$ & $2.08 \pm 0.17 \mathrm{~cd}$ & $12.5 \pm 0.4 \mathrm{c}$ & $2.04 \pm 0.041 \mathrm{c}$ & $21.03 \pm 0.6 \mathrm{c}$ & $3.7 \pm 0.09 \mathrm{bc}$ \\
\hline & $\mathrm{MS}+\mathrm{MW}$ & $13.06 \pm 0.2 \mathrm{ab}$ & $2.3 \pm 0.06 \mathrm{abc}$ & $13.8 \pm 0.45 \mathrm{ab}$ & $2.1 \pm 0.2 \mathrm{bc}$ & $22.8 \pm 0.9 b$ & $3.8 \pm 0.08 \mathrm{~b}$ \\
\hline & $\mathrm{SS}+\mathrm{NW}$ & $11.5 \pm 0.7 \mathrm{~b}$ & $1.95 \pm 0.15 \mathrm{~d}$ & $12.3 \pm 0.62 \mathrm{c}$ & $1.94 \pm 0.08 \mathrm{c}$ & $18.53 \pm 1 \mathrm{~d}$ & $3.42 \pm 0.02 \mathrm{~d}$ \\
\hline & $\mathrm{SS}+\mathrm{MW}$ & $12.6 \pm 1.05 \mathrm{ab}$ & $2.27 \pm 0.29 b c$ & $13.1 \pm 0.4 \mathrm{bc}$ & $1.97 \pm 0.03 \mathrm{c}$ & $20.76 \pm 1.05 \mathrm{c}$ & $3.65 \pm 0.03 \mathrm{c}$ \\
\hline \multirow{6}{*}{$\begin{array}{l}\text { Moringa } \\
\text { peregrina }\end{array}$} & Control+NW & $15 \pm 0.65 b c$ & $2.81 \pm 0.09 \mathrm{a}$ & $1.13 \pm 0.05 \mathrm{bc}$ & $0.31 \pm 0.01 \mathrm{~cd}$ & $6.16 \pm 0.6 b c$ & $1.55 \pm 0.14 \mathrm{bc}$ \\
\hline & Control+MW & $17.1 \pm 1.05 \mathrm{a}$ & $3 \pm 0.24 \mathrm{a}$ & $1.7 \pm 0.4 \mathrm{a}$ & $0.45 \pm 0.05 \mathrm{a}$ & $7.1 \pm 0.4$ & $1.7 \pm 0.1 \mathrm{a}$ \\
\hline & $\mathrm{MS}+\mathrm{NW}$ & $14.2 \pm 0.6 \mathrm{~cd}$ & $2.3 \pm 0.05 b$ & $1.14 \pm 0.04 \mathrm{bc}$ & $0.33 \pm 0.04 \mathrm{bc}$ & $5.73 \pm 0.3 \mathrm{~cd}$ & $1.48 \pm 0.03 \mathrm{c}$ \\
\hline & $\mathrm{MS}+\mathrm{MW}$ & $15.8 \pm 0.5 \mathrm{ab}$ & $2.85 \pm 0.1 \mathrm{a}$ & $1.36 \pm 0.06 \mathrm{~b}$ & $0.37 \pm 0.04 \mathrm{~b}$ & $6.6 \pm 0.3 \mathrm{ab}$ & $1.62 \pm 0.02 \mathrm{ab}$ \\
\hline & $\mathrm{SS}+\mathrm{NW}$ & $13.16 \pm 0.66 \mathrm{~d}$ & $2.4 \pm 0.26 \mathrm{~b}$ & $1.02 \pm 0.02 \mathrm{c}$ & $0.23 \pm 0.005 \mathrm{e}$ & $5.16 \pm 0.4 \mathrm{~d}$ & $1.46 \pm 0.03 \mathrm{c}$ \\
\hline & $\mathrm{SS}+\mathrm{MW}$ & $15.2 \pm 0.6 b c$ & $2.76 \pm 0.1 \mathrm{a}$ & $1.09 \pm 0.01 b c$ & $0.27 \pm 0.01 \mathrm{de}$ & $5.83 \pm 0.4 \mathrm{bcd}$ & $1.55 \pm 0.05 \mathrm{bc}$ \\
\hline
\end{tabular}

Dissimilar letters within mean and between columns are significantly different at $\mathrm{p} \leq 0.05$ level of significance by applying Fisher's LSD Test. 


\section{Ion Content}

The plant samples (root, shoot, and leaves) were prepared using Humpheric's (1956) method [33]. The plant samples were dried and crushed into fine powder. The fine powder from each sample was taken into a digestion tube and $1 \mathrm{ml}$ sulphuric acid $\left(\mathrm{H}_{2} \mathrm{SO}_{4}\right)$ was added. The samples were transferred to a sand heater for 15-20 min until a dark color appeared. Each of the digestion tubes were cooled. We added one ml mixture of sulphuric acid $\left(\mathrm{H}_{2} \mathrm{SO}_{4}\right)$ and percloric acid $\left(\mathrm{HCLO}_{4}\right)$ (1:1) and heated for 30-40 min. Distilled water was added up to $100 \mathrm{ml}$ into each sample containing tubes until transparent colour occurred. The samples were finally transferred to an optima ICP-OECS machine (PerkinElmer Inc., UK) to determine ion.
Statistical Analysis

Analysis of variance (ANOVA) and the mean differences of data were tested by Fisher's LSD test using Minitab (17) statistical software. The differences between the data at $\mathrm{P} \leq 0.05$ were regarded as significant.

\section{Results}

\section{Growth Parameters}

In the present study we observed that magnetic water treatment (MWT) had a statistically significant effect in terms of plant height $(M$. oleifera and
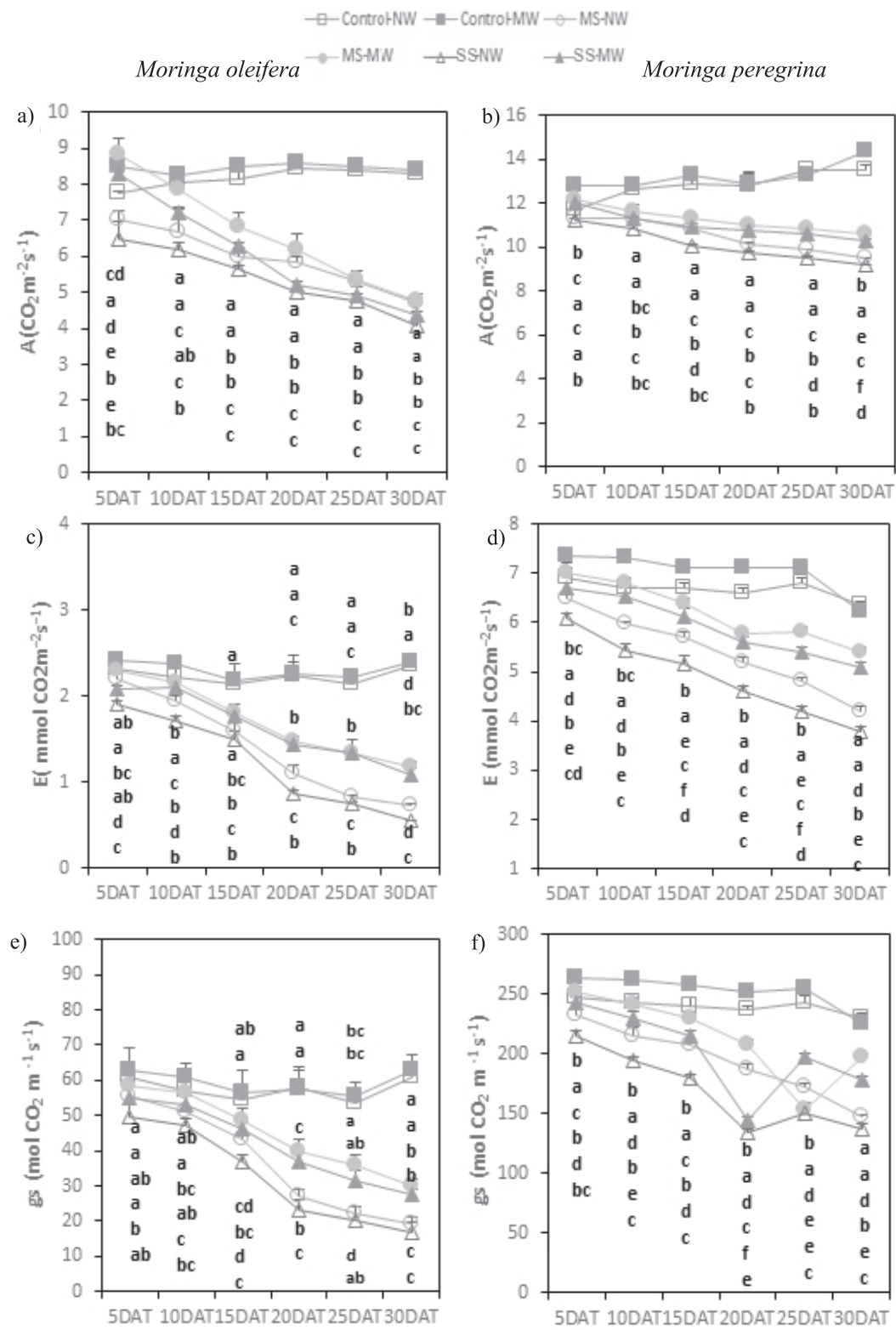

Fig. 3. Effect of magnetic water on assimilation, A; (a, b), transpiration, E; (c, d), and stomatal conductance, gs ;(e, f), of the two Moringa species (Moringa oleifera, Moringa peregrina) under drought stress at 5 to 30 days after treatment; vertical bars represent standard deviation, and different letters above and below the series denote statistically significant $(\mathrm{P} \leq 0.05)$ differences among treatments. 


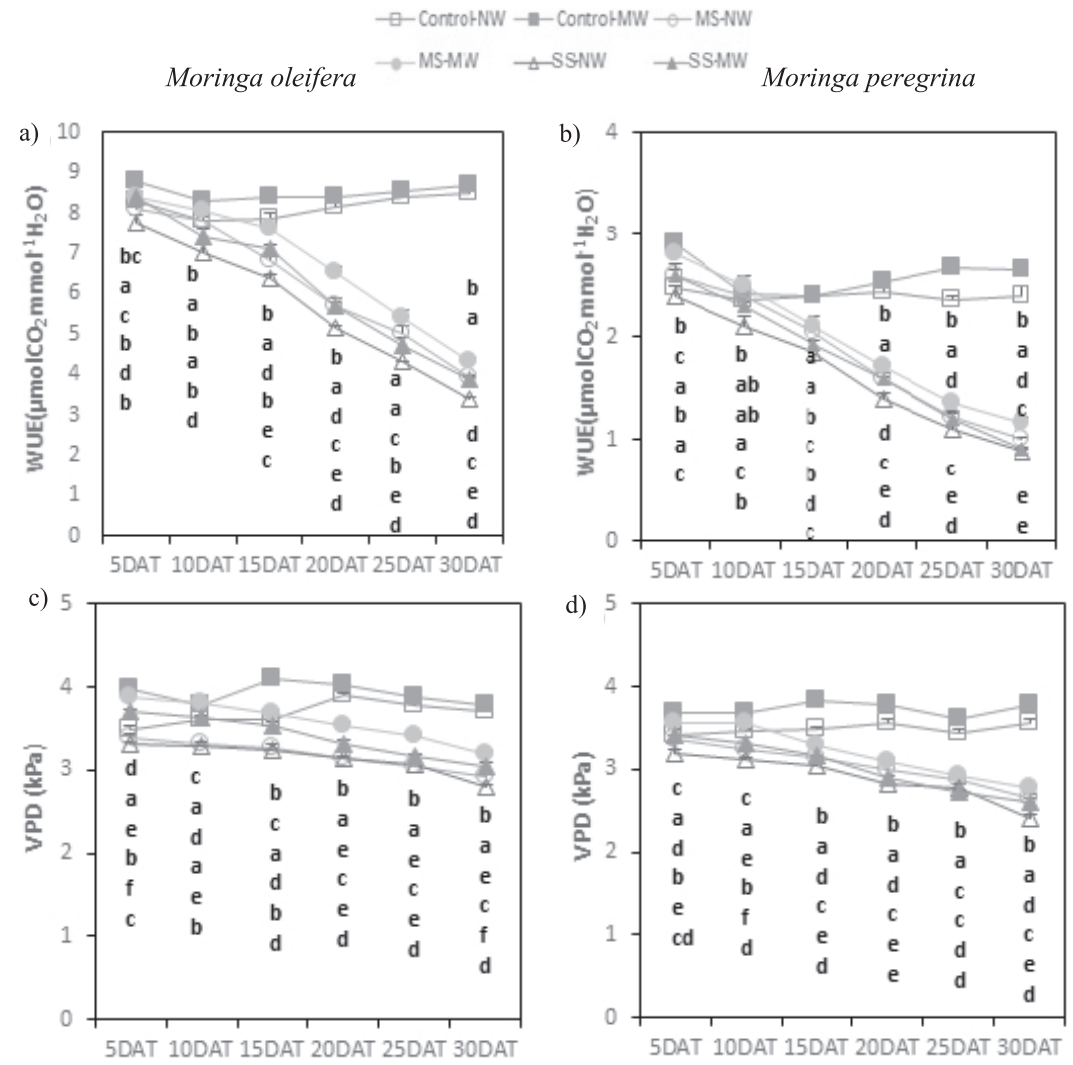

Fig. 4. Water use efficiency, WUE; (a, b), and vapour pressure deficit, VPD; (c, d) of the two Moringa species (Moringa oleifera, MO; Moringa peregrina, MP) under drought stress at 5 to 30 days after treatment; vertical bars represent standard deviation, and different letters above and below the series denote statistically significant $(\mathrm{P} \leq 0.05)$ differences among treatments.

M. peregrina) measured at 5 DAT to 30 DAT (Fig. 1a, b). Drought-induced stress retarded plant height, internode distances, and leaflet number (Fig. 1a-f), respectively.
The application of MWT to the drought stress seedlings significantly restored plant growth compared to the seedlings treated with normal water alone. In addition,

Table 2. Effect of magnetic water on chlorophyll content under different levels of drought stress ( $\left.\mathrm{mgg}^{-1} \mathrm{FW}\right)$.

\begin{tabular}{|c|c|c|c|c|c|}
\hline \multirow{4}{*}{$\begin{array}{c}\text { Moring } \\
\text { oleifera }\end{array}$} & $\begin{array}{c}\text { Treatment } \\
\text { Combinations }\end{array}$ & Chl a & Chl b & Chl (a+b) & Carotenoids \\
\cline { 2 - 6 } & Control+NW & $0.84 \pm 0.03 \mathrm{~b}$ & $0.61 \pm 0.02 \mathrm{bc}$ & $1.45 \pm 0.05 \mathrm{~b}$ & $0.92 \pm 0.01 \mathrm{c}$ \\
\cline { 2 - 6 } & Control+MW & $0.97 \pm 0.01 \mathrm{a}$ & $0.7 \pm 0.03 \mathrm{a}$ & $1.68 \pm 0.045 \mathrm{a}$ & $1.13 \pm 0.02 \mathrm{a}$ \\
\cline { 2 - 6 } & MS+NW & $0.77 \pm 0.02 \mathrm{c}$ & $0.56 \pm 0.03 \mathrm{~d}$ & $1.33 \pm 0.01 \mathrm{c}$ & $0.84 \pm 0.02 \mathrm{e}$ \\
\cline { 2 - 6 } & MS+MW & $0.87 \pm 0.02 \mathrm{~b}$ & $0.64 \pm 0.03 \mathrm{~b}$ & $1.51 \pm 0.01 \mathrm{~b}$ & $0.98 \pm 0.01 \mathrm{~b}$ \\
\cline { 2 - 6 } & SS+NW & $0.6 \pm 0.05 \mathrm{~d}$ & $0.41 \pm 0.02 \mathrm{e}$ & $1.01 \pm 0.03 \mathrm{~d}$ & $0.7 \pm 0.01 \mathrm{f}$ \\
\hline \multirow{5}{*}{$\begin{array}{c}\text { Moringa } \\
\text { peregrina }\end{array}$} & Control+NW & $1.13 \pm 0.02 \mathrm{c}$ & $0.73 \pm 0.03 \mathrm{bc}$ & $1.86 \pm 0.02 \mathrm{c}$ & $1.23 \pm 0.02 \mathrm{c}$ \\
\cline { 2 - 6 } & Control+MW & $1.3 \pm 0.03 \mathrm{a}$ & $0.85 \pm 0.04 \mathrm{a}$ & $2.16 \pm 0.07 \mathrm{a}$ & $1.41 \pm 0.03 \mathrm{a}$ \\
\cline { 2 - 6 } & MS+NW & $1.09 \pm 0.02 \mathrm{~cd}$ & $0.66 \pm 0.03 \mathrm{~d}$ & $1.75 \pm 0.01 \mathrm{~d}$ & $1.14 \pm 0.03 \mathrm{~d}$ \\
\cline { 2 - 6 } & MS+MW & $1.21 \pm 0.04 \mathrm{~b}$ & $0.77 \pm 0.01 \mathrm{~b}$ & $1.99 \pm 0.05 \mathrm{~b}$ & $1.33 \pm 0.03 \mathrm{~b}$ \\
\cline { 2 - 6 } & SS+NW & $0.88 \pm 0.03 \mathrm{e}$ & $0.54 \pm 0.02 \mathrm{e}$ & $1.42 \pm 0.05 \mathrm{e}$ & $0.97 \pm 0.01 \mathrm{e}$ \\
\hline
\end{tabular}

Dissimilar letters within mean and between columns are significantly different at $\mathrm{p} \leq 0.05$ level of significance by applying Fisher's LSD Test. 


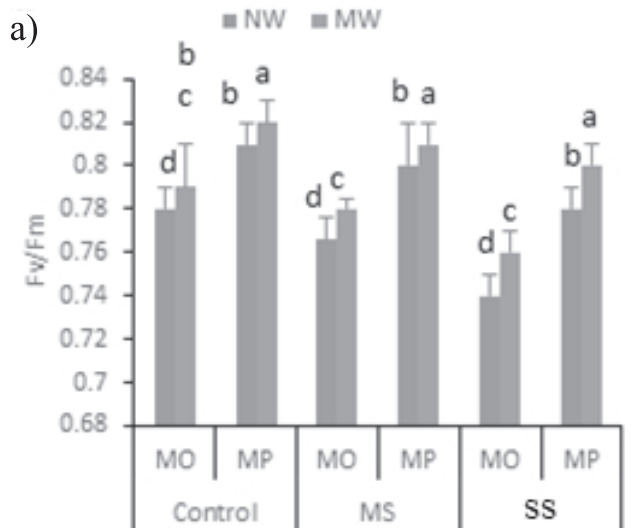

b)

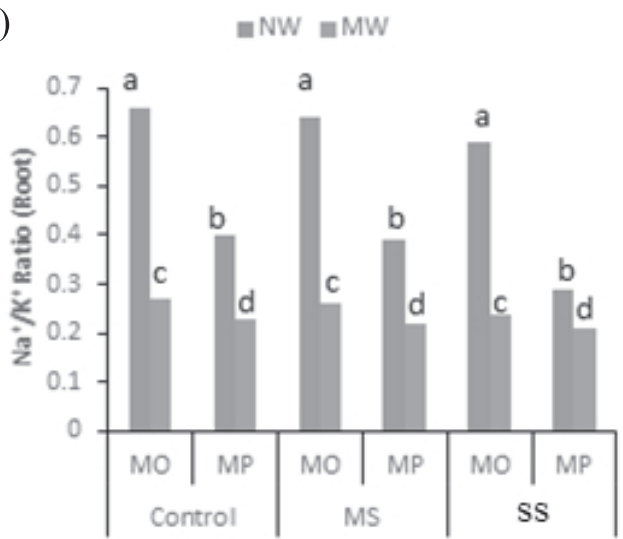

c)

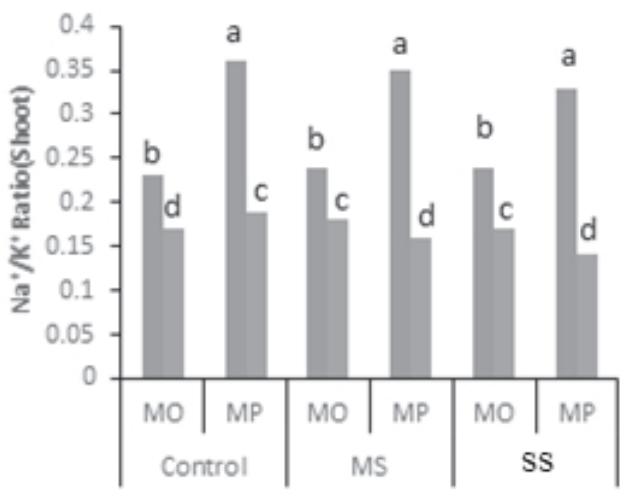

d)

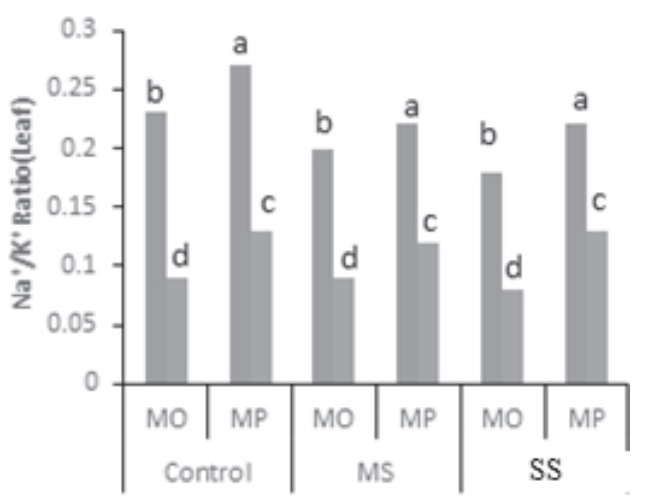

Fig. 5. Effect of magnetic water on the $\mathrm{Fv} / \mathrm{Fm}$ a) and $\mathrm{Na}^{+} / \mathrm{K}^{+}$ ratio in the root b), shoot c), and leaf d) of two Moringa species (Moringa oleifera, MO; Moringa peregrina, MP) under drought stress; dissimilar letters with mean are significantly different at $\mathrm{p} \leq 0.05$ level of significance by applying Fisher's LSD test. morphological components such as root, shoot, leaf, and dry weight were found to be mostly influenced by the MS and SS drought level under normal water conditions and MWT (Table 1).

\section{Leaf Area and Relative Water Content}

Drought stress significantly reduced the leaf area in the M. oleifera seedlings by $3.01 \%$ and $8.4 \%$ respectively, exposed to MS and SS levels as compared to control under normal water conditions. In addition, a significant decrease in leaf area was observed in the M. peregrina seedlings (Fig. 2a). The M. oleifera and $M$. peregrina seedlings exposed to drought stress (MS, SS) showed $4.85 \%, 7.4 \%$ and $3.71 \%, 6.1 \%$ decrease in RWC as compared to control seedlings under normal water conditions (Fig. 2b).

\section{Leaf Gas Exchange}

Data regarding the leaf gas exchange traits of Moringa species were significantly affected under drought stress conditions. Assimilation, transpiration, and stomatal conductance rates were high in Moringa oleifera and Moringa peregrina at 5DAT to 30 DAT (Fig. 3a-f) under $100 \%$ field capacity. At 30 DAT, drought stress caused a significant decrease in transpiration in $M$. oleifera and $M$. peregrina by $68.2 \%, 75.1 \%$, and $29.4 \%, 38.2 \%$ exposed to drought stress (MS, SS) level under normal water conditions. In addition, MWT seedlings showed significantly higher transpiration rate than normal water-treated seedlings. Treating the Moringa seedlings with drought considerably decreased stomatal conductance compared with the control seedlings, whereas MW increased stomatal conductance compared with the seedlings treated with drought alone. Water use efficiency (WUE) and vapor pressure deficit (VPD) significantly affected by the different treatments. Different times of drought stress were also significantly affected by the MW (Fig. 4).

\section{Fv/Fm Ratio}

$\mathrm{F}_{\mathrm{v}} / \mathrm{F}_{\mathrm{m}}$ ratio was decreased in $M$. oleifera and M. peregrina seedlings by $2.56 \%, 5.12 \%$, and $1.2 \%$, $3.84 \%$ exposed to drought stress (MS, SS) level under normal water conditions (Fig. 5a). The $\mathrm{F}_{\mathrm{v}} / \mathrm{F}_{\mathrm{m}}$ ratio increased in the drought-stressed seedlings with MWT.

\section{Chlorophyll Content}

Chlorophyll content (Chl a, Chl b, Chl (a+b), carotenoids) increased under MWT (Table 2). Chl a, $\mathrm{Chl} \mathrm{b}, \mathrm{Chl}(\mathrm{a}+\mathrm{b})$, and carotenoids were decreased in M. oleifera and M. peregrina by $28.57 \%, 32.7 \%, 30.3 \%$, $23.9 \%$ and $22.1 \%, 26.02 \%, 23.6 \%, 21.1 \%$ respectively, exposed to SS under normal water conditions as compared to control seedlings. 
Table 3. Effect of magnetic water on ion content $(\mathrm{mg} / \mathrm{L})$ under drought stress.

\begin{tabular}{|c|c|c|c|c|c|c|}
\hline & Treatment Combination & $\mathrm{Ca}^{2+}(\mathrm{mg} / \mathrm{L})$ & $\mathrm{Mg}^{2+}(\mathrm{mg} / \mathrm{L})$ & $\mathrm{Mn}^{2+}(\mathrm{mg} / \mathrm{L})$ & $\mathrm{P}^{2+}(\mathrm{mg} / \mathrm{L})$ & $\mathrm{Zn}^{2+}(\mathrm{mg} / \mathrm{L})$ \\
\hline \multicolumn{7}{|c|}{ Root } \\
\hline \multirow{6}{*}{$\begin{array}{c}\text { Moringa } \\
\text { oleifera }\end{array}$} & Control+NW & $24.5 \pm 1.8 \mathrm{bc}$ & $11.3 \pm 1.2 \mathrm{bc}$ & $0.13 \pm 0.02 \mathrm{abc}$ & $18 \pm 1 \mathrm{c}$ & $1.01 \pm 0.01 \mathrm{a}$ \\
\hline & Control+MW & $28.31 \pm 2.19 \mathrm{a}$ & $15.4 \pm 1.15 \mathrm{a}$ & $0.16 \pm 0.015 \mathrm{a}$ & $26 \pm 2 a$ & $1.24 \pm 0.03 b$ \\
\hline & $\mathrm{MS}+\mathrm{NW}$ & $22.34 \pm 1.8 \mathrm{c}$ & $9.27 \pm 1.17 \mathrm{~cd}$ & $0.12 \pm 0.02 b c$ & $16.3 \pm 0.57 \mathrm{c}$ & $0.91 \pm 0.06 \mathrm{~d}$ \\
\hline & $\mathrm{MS}+\mathrm{MW}$ & $26.13 \pm 1.9 \mathrm{ab}$ & $13.41 \pm 1.1 \mathrm{ab}$ & $0.15 \pm 0.02 \mathrm{ab}$ & $23.66 \pm 0.57 b$ & $1.1 \pm 0.02$ \\
\hline & $\mathrm{SS}+\mathrm{NW}$ & $18.2 \pm 1.8 \mathrm{~d}$ & $8.8 \pm 1.19 \mathrm{~d}$ & $0.09 \pm 0.02 \mathrm{c}$ & $13 \pm 1 d$ & $0.85 \pm 0.01 \mathrm{e}$ \\
\hline & $\mathrm{SS}+\mathrm{MW}$ & $22.06 \pm 1.8 \mathrm{c}$ & $12.1 \pm 1.22 b$ & $0.13 \pm 0.02 \mathrm{abc}$ & $22 \pm 1 b$ & $0.97 \pm 0.02 \mathrm{~cd}$ \\
\hline \multirow{6}{*}{$\begin{array}{c}\text { Moringa } \\
\text { peregrina }\end{array}$} & Control+NW & $26 \pm 1.8 \mathrm{ab}$ & $10.01 \pm 1.1 \mathrm{abc}$ & $0.17 \pm 0.01 \mathrm{a}$ & $15 \pm 1 \mathrm{c}$ & $0.75 \pm 0.03 \mathrm{bc}$ \\
\hline & Control+MW & $28.9 \pm 1.9 \mathrm{a}$ & $12.3 \pm 1.2 \mathrm{a}$ & $0.16 \pm 0.01 \mathrm{ab}$ & $23 \pm 1 \mathrm{a}$ & $0.91 \pm 0.05 \mathrm{a}$ \\
\hline & $\mathrm{MS}+\mathrm{NW}$ & $24.3 \pm 1.8 \mathrm{bc}$ & $9.6 \pm 1.2 \mathrm{bc}$ & $0.16 \pm 0.01 \mathrm{ab}$ & $14 \pm 1 \mathrm{~cd}$ & $0.65 \pm 0.02 \mathrm{~d}$ \\
\hline & $\mathrm{MS}+\mathrm{MW}$ & $28.2 \pm 1.2 \mathrm{a}$ & $11.31 \pm 1.17 \mathrm{ab}$ & $0.15 \pm 0.02 \mathrm{ab}$ & $22 \pm 1 \mathrm{ab}$ & $0.8 \pm 0.03 \mathrm{~b}$ \\
\hline & $\mathrm{SS}+\mathrm{NW}$ & $21.2 \pm 1.8 \mathrm{c}$ & $8.8 \pm 1.2 \mathrm{c}$ & $0.13 \pm 0.02 b$ & $12.66 \pm 1.15 \mathrm{~d}$ & $0.55 \pm 0.01 \mathrm{e}$ \\
\hline & $\mathrm{SS}+\mathrm{MW}$ & $23.4 \pm 1.8 \mathrm{bc}$ & $10.41 \pm 1.1 b c$ & $0.13 \pm 0.02 b$ & $21 \pm 1 b$ & $0.7 \pm 0.02 \mathrm{c}$ \\
\hline \multicolumn{7}{|c|}{ Shoot } \\
\hline \multirow{6}{*}{$\begin{array}{c}\text { Moringa } \\
\text { oleifera }\end{array}$} & Control+NW & $34.2 \pm 2.5 \mathrm{~cd}$ & $16.5 \pm 1.14 \mathrm{a}$ & $0.19 \pm 0.06 \mathrm{a}$ & $20 \pm 1 \mathrm{c}$ & $0.97 \pm 0.02 \mathrm{~b}$ \\
\hline & Control+MW & $40.5 \pm 2 \mathrm{a}$ & $17.22 \pm 1.17 \mathrm{a}$ & $0.18 \pm 0.05 \mathrm{a}$ & $27.66 \pm 1.5 \mathrm{a}$ & $1.03 \pm 0.02 \mathrm{a}$ \\
\hline & $\mathrm{MS}+\mathrm{NW}$ & $32.1 \pm 2 \mathrm{de}$ & $12.5 \pm 1.14 \mathrm{~b}$ & $0.18 \pm 0.04 \mathrm{a}$ & $18 \pm 1 \mathrm{c}$ & $0.89 \pm 0.02 \mathrm{c}$ \\
\hline & $\mathrm{MS}+\mathrm{MW}$ & $38.2 \pm 2.51 \mathrm{ab}$ & $15.3 \pm 1.09 \mathrm{a}$ & $0.18 \pm 0.03 \mathrm{a}$ & $26 \pm 2 \mathrm{ab}$ & $0.95 \pm 0.01 \mathrm{~b}$ \\
\hline & $\mathrm{SS}+\mathrm{NW}$ & $30.2 \pm 2 \mathrm{e}$ & $10.2 \pm 1.14 \mathrm{c}$ & $0.16 \pm 0.05 \mathrm{a}$ & $15.3 \pm 0.57 \mathrm{~d}$ & $0.88 \pm 0.04 \mathrm{c}$ \\
\hline & $\mathrm{SS}+\mathrm{MW}$ & $36.3 \pm 2.05 b c$ & $13.3 \pm 1.17 \mathrm{~b}$ & $0.17 \pm 0.06 \mathrm{a}$ & $23.66 \pm 1.52 b$ & $0.91 \pm 0.01 \mathrm{c}$ \\
\hline \multirow{6}{*}{$\begin{array}{c}\text { Moringa } \\
\text { peregrina }\end{array}$} & Control+NW & $48.4 \pm 2.1 \mathrm{a}$ & $18.24 \pm 1.2 \mathrm{c}$ & $0.3 \pm 0.06 \mathrm{bc}$ & $16.6 \pm 0.57 \mathrm{c}$ & $1.2 \pm 0.03 \mathrm{a}$ \\
\hline & Control+MW & $50.2 \pm 2.1 \mathrm{a}$ & $24.3 \pm 1.14 \mathrm{a}$ & $0.49 \pm 0.06 \mathrm{a}$ & $24.6 \pm 1.52 \mathrm{a}$ & $1.15 \pm 0.02 \mathrm{~b}$ \\
\hline & $\mathrm{MS}+\mathrm{NW}$ & $41.6 \pm 2.51 \mathrm{c}$ & $12.4 \pm 1.14 \mathrm{~d}$ & $0.28 \pm 0.05 \mathrm{c}$ & $16.3 \pm 0.57 \mathrm{~cd}$ & $1.0 \pm 0.01$ \\
\hline & $\mathrm{MS}+\mathrm{MW}$ & $46.5 \pm 2 \mathrm{ab}$ & $22.4 \pm 0.97 \mathrm{~b}$ & $0.45 \pm 0.06 \mathrm{~b}$ & $22 \pm 1 b$ & $1.03 \pm 0.02 \mathrm{c}$ \\
\hline & $\mathrm{SS}+\mathrm{NW}$ & $40.2 \pm 2.07 \mathrm{c}$ & $10.4 \pm 1.14 \mathrm{e}$ & $0.26 \pm 0.05 \mathrm{c}$ & $14 \pm 2 d$ & $0.91 \pm 0.015 \mathrm{e}$ \\
\hline & $\mathrm{SS}+\mathrm{MW}$ & $43.2 \pm 2.2 b c$ & $20.9 \pm 1.07 \mathrm{~b}$ & $0.4 \pm 0.06 \mathrm{ab}$ & $21.3 \pm 1.52 b$ & $0.96 \pm 0.01 \mathrm{~d}$ \\
\hline \multicolumn{7}{|c|}{ Leaf } \\
\hline \multirow{6}{*}{$\begin{array}{c}\text { Moringa } \\
\text { oleifera }\end{array}$} & Control+NW & $62.4 \pm 2.1 \mathrm{~cd}$ & $18 \pm 1.7 \mathrm{c}$ & $0.6 \pm 0.08 \mathrm{ab}$ & $26.3 \pm 1.15 \mathrm{c}$ & $0.76 \pm 0.02 \mathrm{c}$ \\
\hline & Control+MW & $70.5 \pm 2.18 \mathrm{a}$ & $25.96 \pm 1.6 \mathrm{a}$ & $0.7 \pm 0.07 \mathrm{a}$ & $36 \pm 1 \mathrm{a}$ & $0.89 \pm 0.01 \mathrm{a}$ \\
\hline & $\mathrm{MS}+\mathrm{NW}$ & $59.3 \pm 2.1 d$ & $15.2 \pm 1.75 \mathrm{~cd}$ & $0.58 \pm 0.08 \mathrm{ab}$ & $25 \pm 1 \mathrm{~cd}$ & $0.73 \pm 0.02 \mathrm{~d}$ \\
\hline & $\mathrm{MS}+\mathrm{MW}$ & $66.33 \pm 2.008 b$ & $24.1 \pm 1.7 \mathrm{ab}$ & $0.67 \pm 0.08 \mathrm{a}$ & $35.33 \pm 1.5 \mathrm{ab}$ & $0.79 \pm 0.015 \mathrm{~b}$ \\
\hline & $\mathrm{SS}+\mathrm{NW}$ & $55.3 \pm 2.1 \mathrm{e}$ & $13.1 \pm 1.65 \mathrm{~d}$ & $0.48 \pm 0.08 b$ & $22.3 \pm 2.08 \mathrm{~d}$ & $0.62 \pm 0.005 \mathrm{e}$ \\
\hline & $\mathrm{SS}+\mathrm{MW}$ & $64.3 \pm 2.1 b c$ & $22.03 \pm 1.6 b$ & $0.58 \pm 0.08 \mathrm{ab}$ & $33 \pm 2.64 b$ & $0.72 \pm 0.01 \mathrm{~d}$ \\
\hline \multirow{6}{*}{$\begin{array}{c}\text { Moringa } \\
\text { peregrina }\end{array}$} & Control+NW & $70.4 \pm 2.1 \mathrm{~d}$ & $19.8 \pm 1.7 \mathrm{c}$ & $0.4 \pm 0.07 \mathrm{a}$ & $20.33 \pm 0.57 b$ & $0.71 \pm 0.02 \mathrm{c}$ \\
\hline & Control+MW & $102.3 \pm 2.1 \mathrm{a}$ & $27.9 \pm 1.65 \mathrm{a}$ & $0.4 \pm 0.08 \mathrm{a}$ & $22.6 \pm 1.52 \mathrm{a}$ & $0.78 \pm 0.02 \mathrm{a}$ \\
\hline & $\mathrm{MS}+\mathrm{NW}$ & $65.5 \pm 2.53 \mathrm{e}$ & $14.3 \pm 1.75 \mathrm{~d}$ & $0.35 \pm 0.082 \mathrm{a}$ & $18 \pm 1 \mathrm{~cd}$ & $0.73 \pm 0.015 b$ \\
\hline & $\mathrm{MS}+\mathrm{MW}$ & $90.5 \pm 2.1 b$ & $25.5 \pm 1.65 \mathrm{ab}$ & $0.38 \pm 0.081 \mathrm{a}$ & $19.33 \pm 0.57 b c$ & $0.74 \pm 0.01 \mathrm{~b}$ \\
\hline & $\mathrm{SS}+\mathrm{NW}$ & $60.6 \pm 2.1 \mathrm{f}$ & $11.9 \pm 1.4 \mathrm{~d}$ & $0.3 \pm 0.08 \mathrm{a}$ & $16.6 \pm 0.57 \mathrm{~d}$ & $0.65 \pm 0.01 \mathrm{~d}$ \\
\hline & $\mathrm{SS}+\mathrm{MW}$ & $85.2 \pm 2.05 \mathrm{c}$ & $23 \pm 1.7 b$ & $0.34 \pm 0.06 \mathrm{a}$ & $17.6 \pm 1.52 \mathrm{~cd}$ & $0.71 \pm 0.01 \mathrm{c}$ \\
\hline
\end{tabular}

Dissimilar letters within mean and between columns are significantly different at $\mathrm{p} \leq 0.05$ level of significance by applying Fisher's LSD Test. 


\section{Ion Content}

Ion content $\left(\mathrm{Ca}^{2+}, \mathrm{Mg}^{2+}, \mathrm{Cu}^{2+}, \mathrm{Fe}^{2+}, \mathrm{Mn}^{2+}\right)$ in the root, shoot, and leaf decreased under drought stress (Table 3). The stress induced by drought decreased $\mathrm{Ca}$, $\mathrm{Mg}, \mathrm{Mn}, \mathrm{P}$, and $\mathrm{Zn}$ in the shoots of Moringa oleifera and Moringa peregrina by $11.69 \%, 38.18 \%, 15.7 \%$, $23.5 \%, 9.27 \%$ and $16.9 \%, 42.85 \%, 13.3 \%, 15.6 \%$, $24.1 \%$ respectively, exposed to SS under normal water conditions. However, MW improved the nutrient content in Moringa oleifera and Moringa peregrina. The $\mathrm{Na}^{+} / \mathrm{K}^{+}$ratio increased in the roots, shoot, and leaf in the drought-stressed seedlings and decreased with MW application (Fig. 5b-d).

\section{Discussion}

Drought stress is known to affect the physiology and phenology of plants [30,34]. In our study, plant height, internode distances, and leaflet number were decreased under MS and SS levels (Fig. 1), and significant improvement was found under MWT during drought stress, possibly by improving cell division and cell expansion. The higher leaf area, FW, and DW was observed under MWT, which might be due to the enhancing the photosynthesis rate. These results were in agreement with Souza et al. (2006) [35], who suggested that DW significantly increased under MWtreated seedlings as compared to control. In corn plants, MW alleviated the drought-induced adverse effects on the growth of the plant [36]. Moringa seedlings with drought exhibited lower RWC (Fig. 2b), which indicated that drought stress induced water imbalance and osmotic stress. Magnetic water restored the water loss by increasing the RWC in the drought-affected seedlings.

Our study suggested that assimilation (A), transpiration rate (E), stomatal conductance (gs), water use efficiency (WUE), and vapour pressure deficit (VPD) were increased in both of the Moringa species under MWT. Similar results were observed in Zea mays under mild stress conditions with MWT [37]. The photosynthesis rate improving in Moringa may be due to the improvement of chlorophyll a and photochemical quenching and non-photochemical quenching.

Fluorescence parameter $\mathrm{Fv} / \mathrm{Fm}$ was used to detect stress in plants [38], and our data showed that $\mathrm{Fv} / \mathrm{Fm}$ was decreased due to the adverse effects of drought on the M. oleifera and M. peregrina. The photosystem II damage might be responsible for the reduction of $\mathrm{Fv} / \mathrm{Fm}$. However, Fv/Fm ratio was found to be higher in both Moringa species under MWT. Magnetic water probably helps to alter the photochemical efficiency of photosystem II in the two Moringa species. In soybean plants, higher chlorophyll fluorescence yield [39] was found under MWT, which supported our study outcome.

Our data showed that the Chl a, Chl b Chl(a+b) and carotenoid contents were significantly decreased during drought stress. The chlorophyll content was increased in the leaves of Moringa seedlings under MWT (Table 2). This result is supported by previous studies [40-44] in which, MW enhanced the chlorophyll content in soybean and maize leaves. Anand et al. (2012) [45] reported that the chlorophyll content was increased in corn plants under MW during abiotic stress conditions.

The data showed that the ion content $\left(\mathrm{Ca}^{2+}, \mathrm{Mg}^{2+}\right.$, $\left.\mathrm{Cu}^{2+}, \mathrm{Fe}^{2+}, \mathrm{Mn}^{2+}\right)$ decreased under drought stress conditions (Table 3). MW helped to increase the ion content and this result was supported by previous studies [46]. We have observed that the $\mathrm{Na}^{+}$increased in the roots and shoots of the Moringa species under drought stress. A higher $\mathrm{Na}^{+} / \mathrm{K}^{+}$ratio was found in the Moringa seedlings due to the higher accumulation of $\mathrm{Na}^{+}$, and similar results were found in the rice seedlings under salt stress [47]. The $\mathrm{Ca}^{2+}$ content was decreased, which might be due to the displacement of $\mathrm{Ca}^{2+}$ by $\mathrm{Na}^{+}$.

\section{Conclusions}

We observed that drought stress has significant effects on growth parameters as well as physiological characteristics of the Moringa species. MW assisted in alleviating the drought stress-induced adverse effects on growth, leaf gas exchange, $\mathrm{Fv} / \mathrm{Fm}$ ratio, and chlorophyll and ion contents of the Moringa species. This study may help to understand some adaptive mechanisms developed by Moringa species with MWT.

\section{Abbreviations}

MWT, magnetic water treatment; FC, field capacity; MS, moderate drought stress; SS, severe drought stress; WUE, water use efficiency; RWC, relative water content.

\section{Acknowledgements}

We are very thankful to the dean of scientific research at King Abdulaziz University for providing research funds.

\section{Conflict of Interest}

The authors declare no conflict of interest.

\section{References}

1. CECCARELlI S., GRANDO S., MAATOUGUI M., MICHAEL M., SLASH M., HAGHPARAST R., RAHMANIAN M., TAHERI A., AL-YASSIN A., BENBELKACEM A., LABDI M., MIMOUN H., NACHIT M. Plant breeding and climate changes. J. Agric. Sci. $\mathbf{1 4 8}$ (6), 627, 2010. 
2. MISHRA A.K., SINGH V.P. A review of drought concepts. J. Hydro. 391 (1-2), 202, 2010.

3. NAHAR K., HASANUZZAMAN M., ALAM M.M., FUJITA M. Glutathione-induced drought stress tolerance in mung bean: coordinated roles of the antioxidant defense and methylglyoxal detoxification systems. AoB PLANTS. 7, plv069, 2015.

4. BEGCY K., MARIANO E.D., GENTILE A., LEMBKE C.G., ZINGARETTI S.M., SOUZA G.M., MENOSSI M. A novel stress-induced sugarcane gene confers tolerance to drought, salt and oxidative stress in transgenic tobacco plants. PLoS One. 7, e44697, 2012.

5. KHAN A., ANWAR Y., HASAN M.M., IQBAL A., ALI M., ALHARBY H.F., HAKEEM K.R., HASANUZZAMAN M. Attenuation of Drought Stress in Brassica Seedlings with exogenous application of $\mathrm{Ca}^{2+}$ and $\mathrm{H}_{2} \mathrm{O}_{2}$. Plants. 6, 20, 2017.

6. MEDEIROS D.B., SILVA E.C.D., NOGUEIRA R.J.M.C., TEIXEIRA M.M., BUCKERIDGE M.S. Physiological limitations in two sugarcane varieties under water suppression and after recovering. Theoretical Exp. Plant Physiol. 25 (3), 213, 2013.

7. SILVA E.C., ALBUQUERQUE M.B., AZEVEDO N.A.D., SILVA J.C.D. Drought and its consequences to plants from individual to ecosystem. In: Şener Akınc1, editor. Responses of organisms to water stress. Tech, Croatia, 1, 17, 2013.

8. RAO K.S., LAXMAN R.H., SHIVASHANKARA R.H. Physiological and Morphological Responses of Horticultural Crops to Abiotic Stresses. In: Srinivasa Rao. Abiotic Stress Physiology of Horticultural Crops. Springer, Delhi, India, 1, 3, 2016.

9. KHALILI M., NAGHAVI M.R. Proteins involved in the molecular mechanisms of plant photosynthesis under drought stress. Int. J. Agric. Biosci. 6 (1), 42, 2017.

10. ZANDALINAS S.I., MITTLER R., BALFAGÓN D., ARBONA V., GÓMEZ-CADENAS A. Plant adaptations to the combination of drought and high temperatures. Physiol. Plantarum. 162 (1), 2, 2017.

11. WANG B., LI Z., ENEJI E., TIAN X., ZHAI Z., LI J., DUAN L. Effects of coronatine on growth, gas exchange traits, chlorophyll content, antioxidant enzymes and lipid peroxidation in Maize (Zea mays L.) seedlings under simulated drought stress. Plant Produc. Sci. 11 (3), 67, 2008.

12. GRAÇA J.P., RODRIGUES F.A., FARIAS J.R.B., OLIVEIRA M.C.N., HOFFMANN-CAMPO C.B., ZINGARETTI S.M. Physiological parameters in sugarcane cultivars submitted to water deficit. Braz. J. Plant Physiol. 22 (3), 189, 2010.

13. ANJUM S.A., TANVEER M., ASHRAF U., HUSSAIN S., SHAHZAD B., KHAN I., WANG L. Effect of progressive drought stress on growth, leaf gas exchange, and antioxidant production in two maize cultivars. Environ. Sci. Pollut. Res. 23 (17), 17132, 2016.

14. NAEEM M., NAEEM M.S., AHMAD R., IHSAN M.Z., ASHRAF M.Y., HUSSAIN Y., FAHAD S. Foliar calcium spray confers drought stress tolerance in maize via modulation of plant growth, water relations, proline content and hydrogen peroxide activity. Arch. Agron. Soil Sci. 64 (1), 116, 2017.

15. PENELlA C., CALATAYUd Á., MELGAR J.C. Ascorbic acid alleviates water stress in young peach trees and improves their performance after rewatering. Front. Plant Sci. 8, 1627, 2017.
16. HASAN M.M., ALHARBY H.F., HAJAR A.S., HAKEEM K.R. Leaf gas exchange, $\mathrm{Fv} / \mathrm{Fm}$ ratio, ion content and growth conditions of the two Moringa species under magnetic water treatment. Pak. J. Bot. 49 (3), 921, 2017.

17. FAKHRI A., BEHROUZ S. Assessment of $\mathrm{SnS}_{2}$ nanoparticles properties for photocatalytic and antibacterial applications. Sol. Energy. 117 ,187, 2015.

18. FAKHRI A., POURMAND M., KHAKPOUR R., BEHROUZ, S. Structural, optical, photoluminescence and antibacterial properties of copper-doped silver sulfide nanoparticles. J. Photochem. Photobiol. B: Biol. 149, 78, 2015.

19. AKHRI A., NEJAD P.A. Antimicrobial, antioxidant and cytotoxic effect of Molybdenum trioxide nanoparticles and application of this for degradation of ketamine under different light illumination. J. Photochem. Photobiol. B: Biol. 159, 211, 2016.

20. FAKHRI A., KAHI D.S. Synthesis and characterization of $\mathrm{MnS}_{2}$ /reduced graphene oxide nanohybrids for with photocatalytic and antibacterial activity. J. Photochem. Photobiol. B: Biol. 166, 259, 2017.

21. MOHAMMADIA S., SOHRABI M., GOLIKAND A.N., FAKHRI A. Preparation and characterization of zinc and copper co-doped $\mathrm{WO}_{3}$ nanoparticles: application in photocatalysis and photobiology. J. Photochem. Photobiol. B:Biol. 161, 217, 2016.

22. FAKHRI A., KHAKPOUR R. Synthesis and characterization of carbon or/and boron-doped $\mathrm{CdS}$ nanoparticles and investigation of optical and photoluminescence properties. J. Lumin. 160, 233, 2015.

23. ALI Y., SAMANEH R., KAVAKEBIAN F. Applications of magnetic water technology in farming and agriculture development: A Review of Recent Advances. Curr. World Environ. 9 (3), 695, 2014.

24. ALADJADJIYAN A. Study of the influence of magnetic field on some biological characteristics of Zea mays. J. Central European Agric. 3 (2), 90, 2002.

25. MAHESHWARI B.L., GREWAL H.S. Magnetic treatment of irrigation water: Its effects on vegetable crop yield and water productivity. Agric. water manag. 96 (8), 1229, 2009.

26. GREWAL H.S., MAHESHWARI B.L. Magnetic treatment of irrigation water and snow pea and chickpea seeds enhances early growth and nutrient contents of seedlings. Bioelectromagnetics. 32 (1), 58, 2011.

27. KUMSSA D.B., JOY E.J.M., YOUNG S.D., ODEE D.W., ANDER E.L., BROADLEY M.R. Variation in the mineral element concentration of Moringa oleifera Lam. and M. stenopetala (Bak. f.) Cuf. role in human nutrition. PLoS One. 12, e0175503, 2017.

28. SALEH N.M., MABROUK M.I., SALEM-BEKHIT M.M., HAFEZ E.H. Challenge of Moringa peregrina Forssk as an antimicrobial agent against multi-drug-resistant Salmonella sp. Biotech. Biotecnol. Equipment. 31 (2), 380, 2017.

29. SELIM A.F.H., EL-NADY M.F. Physio-anatomical responses of drought stressed tomato plants to magnetic field. Acta Astronaut. 69 (7-8), 387, 2011.

30. CANAVAR O., GÖTZ K., ELLMER F., CHMIELEWSKI F.M., KAYNAK M.A. Determination of the relationship between water use efficiency, carbon isotope discrimination and proline in sunflower genotypes under drought stress. Australian J. Crop Sci. 8 (2), 232, 2014.

31. ARNON D.T. Copper enzymes in isolated chloroplasts polyphenol oxidase in Beta vulgaris. Plant Physiol. 24 (1), $15,1949$. 
32. LICHTENTHALER H.K., WELLBURN A.R. Determination of Total Carotenoids and Chlorophylls a and b of Leaf Extracts in Different Solvents. Biochem Soc. Trans. 11 (5), 591, 1983.

33. HUMPHRIES E.C. Mineral components and ash analysis. In: Modern Methods of Plant Analysis. Springer, Germany, 1, 468, 1956.

34. KHAN M.S.A., KARIM M.A., HAQUE M.M., ISLAM M.M., KARIM A.J.M.S., MIAN M.A.K. Influence of salt and water stress on growth and yield of soybean genotypes. Pertanika J. Trop. Agri. Sci. 39 (2), 167, 2016.

35. SOUZA A.D., GARCIA D., SUEIRO L., LICEA L., PORRAS E. Pre-sowing magnetic treatment of tomato seeds: effects on the growth and yield of plants cultivated late in the season. Span. Agric. Res. 3 (1), 113, 2005.

36. JAVED N., ASHRAF M., AKRAM N., AL-QURAINY F. Alleviation of adverse effects of drought stress on growth and some potential physiological attributes in maize (Zea mays) by seed electromagnetic treatment. Photochem. Photobiol. 87 (6), 1354, 2011.

37. ANAND A., NAGARAJAN S., VERMA A., JOSHI D., PATHAK P., BHARDWAJ J. Pre-treatment of seeds with static magnetic field ameliorates soil water stress in seedlings of maize (Zea mays L). Ind. J. Biochem. Biophys. 49 (1), 63, 2012.

38. MAXWELL K., JOHNSON G.N. Chlorophyll fluorescence a practical guide. J. Exp. Bot. 51 (345), 659, 2000.

39. MAFFEI E.M. Magnetic field effects on plant growth, development, and evolution. Front. Plant Sci. 5, 545, 2014.

40. ROCHALSKA M. Influence of frequent magnetic field on chlorophyll content in the leaves of sugar beet plants. Nukleonika. 50 (2), 25, 2005.
41. TURKER M., TEMIRCI C., BATTAL P., EREZ M.E. The effects of an artificial and static magnetic field on plant growth, chlorophyll and phytohormone levels in maize and sunflower plants. Phyton. Ann. Rei. Bot. 46 (2), 271, 2007.

42. RADHAKRISHNAN R., KUMARI B.D.R. Protective role of pulsed magnetic field against salt stress effects in soybean organ culture. Plant Biosystems. 147 (1), 135, 2013.

43. KATARIA S., BAGHEL L., GURUPRASAD K.N. Effect of seed pretreatment by magnetic field on the sensitivity of maize seedlings to ambient ultraviolet radiation (280-400 nm). Int. J. Trop. Agric. 33 (4), 7, 2015.

44. BAGHEL L., KATARIA S., GURUPRASAD K.N. Static magnetic field treatment of seeds improves carbon and nitrogen metabolism under salinity stress in soybean. Bioelectromagnetics. 37 (7), 455, 2016.

45. ANAND A., NAGARAJAN S., VERMA A., JOSHI D., PATHAK P., BHARDWAJ J. Pre-treatment of seeds with static magnetic field ameliorates soil water stress in seedlings of maize (Zea mays L). Ind. J. Biochem. Biophys. 49, 63, 2012.

46. E'ITKEN A., TURAN M. Alternating magnetic field effects on yield and plant nutrient element composition of strawberry (Fragaria x ananassa cv. Camarosa). Acta Agric. Scandinavica, Section B-Soil Plant Sci. 54 (3), 135, 2004.

47. RAHMAN A., NAHAR K., HASANUZZAMAN M., FUJITA M. Calcium Supplementation Improves $\mathrm{Na}^{+} / \mathrm{K}^{+}$ Ratio, Antioxidant Defense and Glyoxalase Systems in Salt-Stressed Rice Seedlings. Front. Plant Sci. 7, 609, 2016. 
